\title{
Measurement of pulmonary oedema in ischaemic heart disease
}

\author{
R. M. McCredie and B. L. Chia ${ }^{1}$ \\ From the Division of Cardiology, The Prince Henry Hospital, Sydney, N.S.W., Australia
}

Pulmonary extravascular fluid volume was measured in 64 patients with ischaemic heart disease at the time of cardiac catheterization using a double indicator dilution technique. In 31 of these patients exercise studies were done. Left ventricular and selective coronary angiography was performed in 43 and the angiographic severity of left ventricular dysfunction and of coronary artery obstructive disease was given a numerical score.

There was no correlation between pulmonary extravascular fluid volume and resting left atrial pressure $(r=0 \cdot 17)$. There was, however, a slight significant correlation between the former and $P_{\overline{L A}}$ on exercise $(r=$ $+0.404, p<0.025)$. If the patients are divided into those with normal $\left(<150 \mathrm{ml} / \mathrm{m}^{2}\right)$ and high extravascular fluid volume ( $>150 \mathrm{ml} / \mathrm{m}^{2}$ ), the high group had a slightly but significantly higher mean left atrial pressure at rest, but still within normal limits. Mean $P_{\overline{L A}}$ in the normal group was normal both at rest and on exercise, but in the high group, mean $P_{\overline{L A}}$ became much higher on exercise. Pulmonary extravascular fluid volume was also significantly related to the left ventricular end-diastolic pressure after left ventricular angiography $(r=$ $+0.333, P<0.05)$ and with the angiographic grading of left ventricular dysfunction $(r=+0.424, P<0.005)$. It was not related to the angiographic severity of coronary artery obstructive disease.

Raised pulmonary extravascular fluid volume has been widely accepted as an index of pulmonary oedema of which left atrial pressure is an important determinant. Pulmonary extravascular fluid volume is related to resting $P_{\overline{L A}}$ in patients with valvular heart disease. In ischaemic heart disease patients this relation is only really apparent when the ventricle is stressed as by exercise. It is suggested that in ischaemic heart disease patients with diminished myocardial reserve, normal daily activities result in frequent rise in left atrial pressure which is reflected by raised values of pulmonary extravascular volume. This remains chronically raised even though left atrial pressure returns to normal at rest. Thus pulmonary extravascular fluid volume may be useful as an indicator for left atrial ventricular failure in ischaemic heart disease.

Measurements of pulmonary extravascular fluid volume by the diffusible indicator method of Chinard and Enns (1954) have previously been reported in patients with valvular heart disease (McCredie, 1967). A good correlation between left atrial pressure at rest and pulmonary extravascular fluid volume was demonstrated, and it was inferred that left atrial pressure was a major determinant of pulmonary oedema as measured by extravascular fluid volume.

We have applied this method to 64 patients with ischaemic heart disease referred for diagnostic assessment, which included cardiac catheterization and in 43 cases selective coronary angio-

Received 14 May 1973.

1 Present address: Department of Medicine (Unit I), University of Singapore, Outram Road General Hospital, Singapore 3 . graphy and left ventriculography. In the first 24 patients only resting haemodynamic measurements were made and it became apparent that the relation between pulmonary extravascular fluid volume and resting left atrial pressure $\left(P_{\overline{\mathrm{LA}}}\right)$ seen in patients with valvular heart disease did not exist in this group. Subsequently all patients in whom resting $P_{\overline{L A}}$ was normal were subjected to a standard exercise test. The pulmonary extravascular fluid volume was also related to the angiographic assessment of the severity of coronary artery disease and left ventricular dysfunction.

\section{Subjects and methods}

Sixty-four patients with a clinical diagnosis of ischaemic heart disease confirmed by electrocardiogram were referred for study. Right heart catheterization was per- 
formed, an arterial needle was placed in the brachial artery, and resting measurements of pulmonary extravascular fluid volume, pulmonary arterial pressure $\left(P_{\overline{L A}}\right)$, indirect left atrial (pulmonary wedge) pressure $\left(\mathrm{P}_{\overline{\mathrm{LA}}}\right)$, and cardiac output (CO) were made in all. In 31 patients, an exercise test was performed - straight leg raising at a constant rate of $40 / \mathrm{min}$ for 5 minutes or until mean $P_{\overline{L A}}$ rose to $30 \mathrm{mmHg}$, at which time, with the subject still exercising, measurements of $\mathrm{P}_{\overline{\mathrm{LA}}}, \mathrm{P}_{\overline{\mathrm{PA}}}$, and $\mathrm{CO}$ were repeated.

Normal data for $\mathrm{P}_{\overline{\mathrm{LA}}}$ in response to this exercise test were obtained from ro patients referred for evaluation of chest pain who had no clinical, electrocardiographic, or radiological abnormality, and who were found to have normal haemodynamics and angiographically normal coronary arteries and left ventricles.

In 43 of these patients, selective coronary arteriography was performed by the Sones technique via a right brachial arteriotomy. Left ventricular pressure was measured before and after left ventricular cineangiography with a pressure injection of $50 \mathrm{ml} 76$ per cent Urografin. The angiographic severity of coronary artery obstructive disease was assigned a numerical score as outlined by Gensini and Buonanno (I968) and the angiographic severity of left ventricular dysfunction was graded 0 to 4 . All studies were done after premedication with papaveretum 10 to $20 \mathrm{mg}$ and only local anaesthesia was used.

The double indicator dilution technique for the measurement of pulmonary extravascular fluid volume as described in detail previously (McCredie, 1967) has been slightly modified. A mixture of radioiodinated serum albumin (RISA) and tritiated water (THO) containing approximately $2.5 \mu \mathrm{c}{ }^{125} \mathrm{I}$ and $50 \mu \mathrm{c}{ }^{3} \mathrm{H}$ was injected into the right side of the heart proximal to the pulmonary artery. Blood samples ( $1 \mathrm{ml}$ ) were taken at one-second intervals with a motor driven roller pump. ${ }^{1}$ A $0.5 \mathrm{ml}$ aliquot of each sample was transferred to a I5 $\mathrm{ml}$ test tube and ${ }^{125} \mathrm{I}$ activity determined by counting for Io minutes in a Packard auto-gamma counter. Standards made by diluting the injectate with known volumes of the patient's blood were counted in the same way. The water content in each sample was then extracted under vacuum and transferred to a scintillation mixture made up of $4 \mathrm{~g}$ of 2:5-diphenyl-oxazole and $0.154 \mathrm{~g}$ of $\mathrm{I}: 4 \mathrm{di}$ (2-( 5 phenyl-oxazolyl)) benzene in $\mathrm{I}$ litre of toluene. These samples were counted for Io minutes in a Packard Tricarb Model 3I4 EX liquid scintillation spectrometer.

Counting rates for tritium and iodine were similar to those previously recorded. From replicate analyses, the standard errors for both ${ }^{3} \mathrm{H}$ and ${ }^{125} \mathrm{I}$ counts were less than \pm 2.5 per cent. Time concentration curves for both RISA and THO were plotted on semilogarithmic paper. Cardiac output and mean transit times were determined for each curve (Hamilton et al., 1932) and pulmonary extravascular fluid volume was calculated as the product of the mean cardiac output from the RISA and THO curves and the difference between mean transit times.

1 Manostat Corp., New York.

\section{Results}

Comparison between cardiac outputs determined independently from the RISA and THO curves showed that the mean value from the THO curve was 5.1 per cent higher than that obtained from the RISA curves, and this difference was significant $(\mathrm{P}<0.00 \mathrm{I})$.

The mean value of pulmonary extravascular fluid volume in all patients with ischaemic heart disease was $172 \pm 85 \mathrm{ml} / \mathrm{m}^{2}$ (mean $\pm \mathrm{SD}$ ), and this was significantly higher than the normal range previously reported of $107 \pm 22.4 \mathrm{ml} / \mathrm{m}^{2}(P<0.001)$. There was no correlation between pulmonary extravascular fluid volume and arterial oxygen saturation $(r=$ $-0.05)$.

The relation of extravascular fluid volume to resting left atrial pressure is shown in Fig. I. There was no significant correlation $(r=0.17,0.10$ $<P<0.15)$. In general those with a high $\mathrm{P}_{\overline{\mathrm{LA}}}$ had a high pulmonary extravascular fluid volume but many with a high volume had a normal resting $\mathrm{P}_{\overline{\mathrm{LA}} \text {. }}$

Comparison of fluid volume with $\mathrm{P}_{\overline{\mathrm{LA}}}$ during exercise in 31 patients who had a normal resting $P_{\overline{\mathrm{LA}}}$ is shown in Fig. 2. There is in this case a significant correlation between the two $(r=+0.404$, $P<0.025)$.

In Fig. 3 all 64 patients are divided into those with a normal pulmonary extravascular fluid volume $\left(<150 \mathrm{ml} / \mathrm{m}^{2}\right)$ and those with a high volume

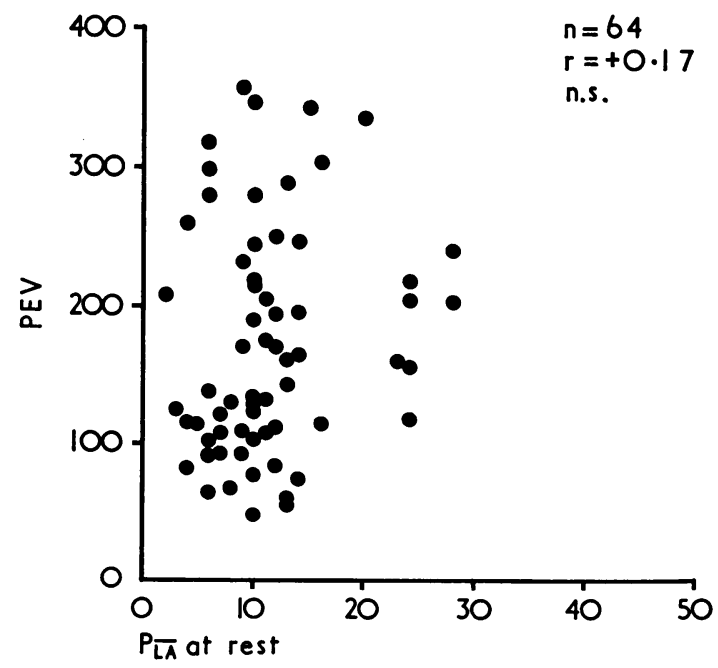

FIG. I Relation of pulmonary extravascular fluid volume to resting mean left atrial pressure in 64 patients with ischaemic heart disease. No significant correlation $(0 \cdot 10<P<0 \cdot 15)$. 


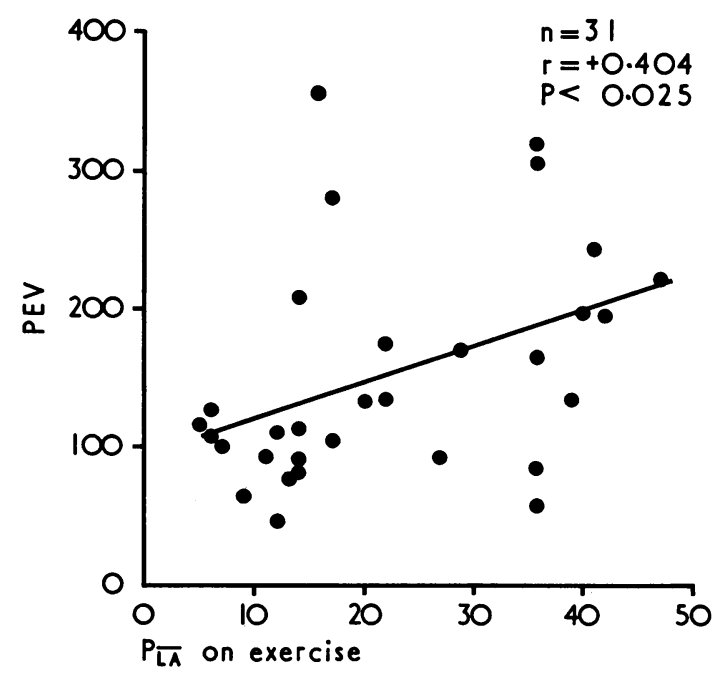

FIG. 2 Relation of pulmonary extravascular fluid volume to mean left atrial pressure during exercise in $3 I$ patients with normal resting left atrial pressures. The correlation is significant $(P<0.025)$.

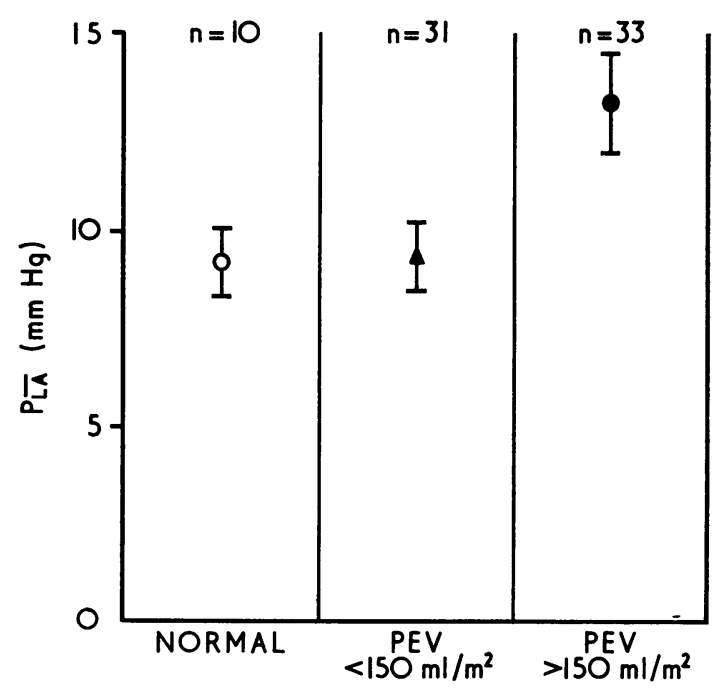

FIG. 3 Comparison of resting mean left atrial pressure (mean $\pm S E$ ) in 10 normal subjects, 3I ischaemic heart disease patients with normal pulmonary extravascular fluid volume (no significant difference), and 33 ischaemic heart disease patients with abnormally high pulmonary extravascular fluid volume. $P_{\overline{\mathrm{LA}}}$ in the latter group is significantly higher than in the normal subjects $(P<0.05)$ and in the normal pulmonary extravascular fluid volume $\operatorname{group}(P<0.01)$.
( $>150 \mathrm{ml} / \mathrm{m}^{2}$ ). The normal group had a normal resting $\mathrm{P}_{\overline{\mathrm{LA}}}$ of $9.5 \mathrm{mmHg}(\mathrm{SE} \pm 0.8)$ compared with the normal in this laboratory of $9.2 \mathrm{mmHg}$ $(S E \pm 0.9)$. The high group had a resting $P_{\overline{L A}}$ of 13.3 $\mathrm{mmHg} \mathrm{SE} \pm \mathrm{r} \cdot 2$, which was significantly higher than the normal $(P<0.05)$ and than the mean for the normal group $(P<0.01)$.

Fig. 4 shows the $P_{\overline{L A}}$ response to exercise in $3 I$ patients with a normal resting $P_{\overline{L A}}$ grouped in the same way. In I9 patients with a normal pulmonary extravascular fluid volume, mean $P_{\overline{\mathrm{LA}}}$ at rest was $8.9 \mathrm{mmHg}(\mathrm{SE} \pm 0.4)$ and rose to a mean value of $16.8 \mathrm{mmHg}(\mathrm{SE} \pm 2.4)$ on exercise, not significantly higher than the normal in this laboratory of 14.8 $\mathrm{mmHg}(\mathrm{SE} \pm 2 \cdot 8)$. The high group of 12 patients had a slightly but significantly higher resting $P_{\overline{L A}}$ of II.2mmHg, $(S E \pm 0.9)(P<0.025)$ but on exercise $P_{\overline{L A}}$ rose to a mean value of $28.8 \mathrm{mmHg}$ (SE \pm 3.2$)$; a highly significant difference from both the normal range and the normal group of patients $(P<0.005)$.

Pulmonary extravascular fluid volume was significantly related to left ventricular end-diastolic pressure after left ventricular angiography $(r=$ $+0.333, P<0.05)$ and to the angiographic grading

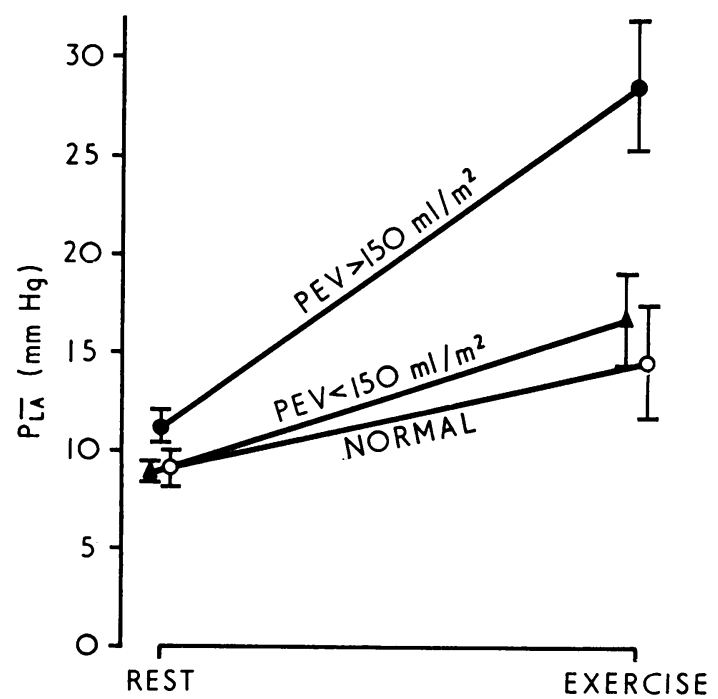

FIG. 4 Left atrial pressure (mean $\pm S E$ ) response to exercise in 10 normal subjects, 19 patients with normal extravascular fluid volume, and I 2 patients with high values. In the latter group $P_{\overline{\mathrm{LA}}}$ at rest was slightly higher than in normal $(P<0.05)$ and in the normal pulmonary extravascular fluid volume group $(P<0.025)$, but was much more significantly higher on exercise $(P<0.005$ for both $)$. 
of left ventricular function $(r=+0.424, P<0.005)$. There was no correlation between extravascular fluid volume and the angiographic score for coronary artery obstructive lesions $(r=+0.120,0.5>$ $\mathrm{P}>0.4)$.

\section{Discussion}

Errors and limitations of the method of measurement of pulmonary extravascular fluid volume have been discussed in detail in the previous report from this laboratory (McCredie, 1967) as well as by others. There is no reason to suppose that any systematic error of the measurement occurs which could influence the present results.

There is a small difference in the cardiac outputs measured individually from the RISA and THO curves. A similar difference has been noted in some but not all previous reports where it has been studied (Ramsey et al., I964; Pearce, Yamashita, and Beazell, 1965; Goresky, Cronin, and Wangel, 1969; Chinard, Perl, and Effros, 1973). This difference represents loss of water from the system during the single passage through the pulmonary capillary bed. However, although statistically significant, it is not of sufficient magnitude to influence these results.

Sutherland, Cade, and Pain (197I) measured pulmonary extravascular fluid volume in 14 patients after acute myocardial infarction and found, in 6 patients with raised levels, a greater degree of hypoxaemia due to venoarterial shunting. In this larger group of patients reported here, none of whom were in the stage of acute or recent infarction, no relation was found between fluid volume and arterial oxygen saturation. As has been pointed out before (McCredie, 1967, 1970), venoarterial shunting can lead to underestimates of the pulmonary extravascular fluid volume. Any systematic error from this source should be associated with lower values in those with arterial hypoxaemia, which did not occur.

In patients with ischaemic heart disease, unlike those with valvular heart disease (McCredie, 1967), no direct correlation was found between pulmonary extravascular fluid volume and mean $\mathrm{P}_{\overline{\mathrm{LA}}}$ at rest (Fig. I), but there is a significant correlation with mean $P_{\overline{L A}}$ on exercise (Fig. 2). It is apparent from Fig. 3 and 4 that ischaemic heart disease patients with a normal pulmonary extravascular fluid volume have, as a group, normal resting $\mathrm{P}_{\overline{\mathrm{LA}}}$ and a normal $P_{\overline{\mathrm{LA}}}$ response to exercise, whereas the group with high levels, though they have a slightly higher resting $\mathrm{P}_{\overline{\mathrm{LA}}}$, have a grossly abnormal $\mathrm{P}_{\overline{\mathrm{LA}}}$ response to exercise. In ischaemic heart disease patients, the relation of high pulmonary extravascular fluid volume to high $\mathrm{P}_{\overline{\mathrm{LA}}}$ is only really apparent under stress.

The difference in the extravascular fluid volumeleft atrial pressure relation in ischaemic and valvular heart disease may be due to the different mechanism of left atrial pressure rise in the two situations, and to the time sequence of fluid accumulation in the lungs. In valvular heart disease, a high left atrial pressure is usually due to mechanical valve malfunction; though aggravated by exercise, it is likely to remain chronically raised at rest as well. In ischaemic heart disease, however, a high $\mathrm{P}_{\overline{\mathrm{LA}}}$ is usually due to left ventricular failure and frequently occurs only under stress, such as exercise or angina. Abnormal rises of $\mathrm{P}_{\overline{\mathrm{LA}}}$ under stress in patients with impaired myocardial function may return to normal when the stress ceases.

Accumulation of extravascular lung water is a relatively slow process. It depends on the balance between a number of interrelated factors. First is the net movement of water from capillaries, which depend on the effective pressure tending to drive water out - the net result of hydrostatic and osmotic forces. The most important variable determining this effective pressure is the hydrostatic pressure within the capillaries, and this is likely to be most influenced by left atrial pressure. Thus change in left atrial pressure is the chief determinant of change in the net movement of water out of capillaries. Extravascular lung water depends secondly on the capacity and distensibility of the extravascular water space, including alveolar-capillary walls and alveoli which can become fluid filled, and thirdly on pulmonary lymphatic drainage.

Experiments in dogs have shown that an acute rise in $\mathrm{P}_{\overline{\mathrm{LA}}}$ leads to a rise in pulmonary extravascular fluid volume only after a considerable time lag: it is not detectable at 15 minutes and only really apparent after an hour (Pearce, Nagano, and Staub, 1964). The time course of the reverse process has not been as well studied, but it is probably reasonable to assume that falls in pulmonary extravascular fluid volume after acute falls in $\mathrm{P}_{\overline{\mathrm{LA}}}$ occur slowly as do the rises.

One could postulate that the lungs act like a sponge into which water flows from the capillaries and seeps out via lymphatic drainage. The input depends mainly on net pressure within capillaries and can be varied rapidly. The substance of the sponge has a considerable capacity for water not only in the material framework but also in the air spaces, and changes in water seeping out occur much more slowly. Though in any equilibrium state, total water input and output must be equal, a faster input from higher driving pressure will result in an increased volume held on the sponge and a 
slower increase in output until it matches the input. Thus rapid changes in $\mathrm{P}_{\overline{\mathrm{LA}}}$ lead to slower changes in total fluid movement and relatively slower changes in pulmonary extravascular fluid volume. It seems likely that the whole complex system adapts itself to the usual state of left atrial pressure, and that measurements of pulmonary extravascular fluid volume are likely to reflect this usual state of $\mathrm{P} \overline{\mathrm{LA}}$, whether constantly raised as in valvular heart disease or intermittently as in ischaemic heart disease.

It has been pointed out that the extravascular fluid volume by this diffusible indicator method does not measure all the water in the lungs. It only measures about 70 per cent of the water content at necropsy of normal dogs' lungs but a considerably higher proportion of the water in oedematous lungs (Pearce et al., 1965). Presumably it only measures the water space in close relation to capillaries and does not include the more distant interstitial spaces related to larger airways and the lymphatic system.

This relation of pulmonary extravascular fluid volume to $\mathrm{P}_{\overline{\mathrm{LA}}}$ during exercise is also similar to the relation between fluid volume and left ventricular end-diastolic pressure after angiography and to the angiographic severity of left ventricular dysfunction. Of these three independent methods of assessing left ventricular failure, all show a good correlation with pulmonary extravascular fluid volume. This supports the idea that this is a useful index of interstitial pulmonary oedema and also that left atrial pressure is an important determinant of extravascular fluid volume in ischaemic heart disease.

Pulmonary extravascular fluid volume was not related to the angiographic severity of coronary artery obstruction; neither was this angiographic score related to left ventricular end-diastolic pressure after ventriculography though there was a correlation with $\mathrm{P}_{\overline{\mathrm{LA}}}$ on exercise. It appears, at least in this series, that the severity of coronary artery disease as assessed angiographically is not closely related to indices of left ventricular failure.

This work was supported in part by grants from the National Heart Foundation of Australia (G571/380) and from Sandoz (Australia). I would like to thank Dr. M. J. Swinburn for his help and Mr. R. Williams, Mrs. H. Dunnett, and Mrs. F. Worner for their technical assistance.

\section{References}

Chinard, F. P., and Enns, T. (1954). Transcapillary pulmonary exchange of water in the dog. American fournal of Physiology, 178, 197.

Chinard, F. P., Perl, W., and Effros, R. M. (1973). Theoretical and practical considerations on the measurement of extravascular lung water. In Central Hemodynamics and Gas Exchange, I97I, p. 57. Ed. by C. Giuntini. Minerva Medica, Torino.

Gensini, G. G., and Buonanno, C. (1968). Coronary arteriography: a study of 100 cases with angiographically proved coronary artery disease. Diseases of the Chest, 54,90.

Goresky, C. A., Cronin, R. F. P., and Wangel, B. E. (1969). Indicator dilution measurements of extravascular water in the lungs. Fournal of Clinical Investigation, 48, 487.

Hamilton, W. F., Moore, J. W., Kinsman, J. M., and Spurling, R. G. (1932). Studies on the circulation. IV. Further analysis of the injection method and of changes in hemodynamics under physiological and pathological conditions. American fournal of Physiology, 99, 534 .

McCredie, R. M. (1967). The measurement of pulmonary edema in valvular heart disease. Circulation, 36, 381.

McCredie, R. M. (1970). Pulmonary oedema in lung disease. British Heart fournal, 32, 66.

Pearce, M. L., Nagano, H., and Staub, N. C. (1964). Acute pulmonary edema due to elevation of pulmonary capillary pressure. Clinical Research, 12, 121.

Pearce, M. L., Yamashita, J., and Beazell, J. (1965). Measurement of pulmonary edema. Circulation Research, 16, 482.

Ramsey, L. H., Puckett, W., Jose, A., and Lacy, W. W. (I964). Pericapillary gas and water distribution volumes of the lung calculated from multiple indicator dilution curves. Circulation Research, 15, 275.

Sutherland, P. W., Cade, J. F., and Pain, M. C. F. (I97I). Pulmonary extravascular fluid volume and hypoxaemia in myocardial infarction. Australian and New Zealand fournal of Medicine, $\mathrm{I}$, $\mathrm{r} 4 \mathrm{I}$.

Requests for reprints to Dr. R M. McCredie, Division of Cardiology, The Prince Henry Hospital, Sydney, N.S.W., Australia. 